\title{
EMBEDDING MODULES OF FINITE HOMOLOGICAL DIMENSION
}

\author{
SEAN SATHER-WAGSTAFF \\ Mathematics Department, NDSU, Dept \# 2750, PO Box 6050, Fargo, ND 58108-6050 USA \\ e-mail: Sean.Sather-Wagstaff@ndsu.edu
}

(Received 26 July 2011; revised 14 January 2012; accepted 2 May 2012; first published online 2 August 2012)

\begin{abstract}
This paper builds on work of Hochster and Yao that provides nice embeddings for finitely generated modules of finite G-dimension, finite projective dimension or locally finite injective dimension. We extend these results by providing similar embeddings in the relative setting, that is, for certain modules of finite $\mathrm{G}_{C^{-}}$ dimension, finite $\mathcal{P}_{C}$-projective dimension, locally finite $\mathcal{G} \mathcal{I}_{C}$-injective dimension or locally finite $\mathcal{I}_{C}$-injective dimension where $C$ is a semidualizing module. Along the way, we extend some results for modules of finite homological dimension to modules of locally finite homological dimension in the relative setting.
\end{abstract}

2000 Mathematics Subject Classification. 13D02, 13D05, 13 D07.

1. Introduction. Throughout this note, $R$ is a commutative Noetherian ring with identity. The purpose of this note is to extend some results of Hochster and Yao, beginning with the following, wherein $\underline{z}_{[i]}=z_{1}, \ldots, z_{i}$ and $\mathrm{G}-\operatorname{dim}_{R}(M)$ is the G-dimension of Auslander and Bridger [1, 2]. ${ }^{1}$ Then

THEOREM 1.1 [14, Theorem 2.3]. Let $M$ be a non-zero finitely generated $R$-module.

(1) If $\mathrm{G}-\operatorname{dim}_{R}(M)=r<\infty$, then there are an $R$-regular sequence $\underline{z}=z_{1}, \ldots, z_{r}$, integers $n_{0}, n_{1}, \ldots, n_{r} \geqslant 0$ with $n_{r} \geqslant 1$, and an exact sequence

$$
0 \rightarrow M \rightarrow Z \rightarrow N \rightarrow 0
$$

with $Z=\oplus_{i=0}^{r}\left(R /\left(\underline{z}_{[i]}\right)\right)^{n_{i}}$ and $\mathrm{G}-\operatorname{dim}_{R}(N) \leqslant r$.

(2) If $\operatorname{pd}_{R}(M)=r<\infty$, then there exist an $R$-regular sequence $\underline{z}=z_{1}, \ldots, z_{r}$, integers $n_{0}, n_{1}, \ldots, n_{r} \geqslant 0$ with $n_{r} \geqslant 1$, and an exact sequence

$$
0 \rightarrow M \rightarrow Z \rightarrow N \rightarrow 0
$$

with $Z=\oplus_{i=0}^{r}\left(R /\left(\underline{z}_{[i]}\right)\right)^{n_{i}}$ and $\operatorname{pd}_{R}(N) \leqslant r$.

The point of Theorem 1.1, as Hochster and Yao indicate, is that the given module of finite G-dimension or projective dimension embeds in a module that obviously has

\footnotetext{
${ }^{1}$ This is also now called 'Gorenstein projective dimension' after the work of Enochs, Jenda and others. However, this is a generalisation of Auslander and Bridger's original notion for non-finitely-generated modules. Since we are focused on finitely generated modules here, we continue with Auslander and Bridger's terminology.
} 
finite projective dimension, with some extra control on the G-dimension or projective dimension of the co-kernel.

The first of our results, stated next, is for modules of finite $\mathrm{G}_{C}$-dimension and modules of finite $\mathcal{P}_{C}$-projective dimension, where $C$ is a semidualizing $R$-module, after Golod [12], Holm and Jørgensen [15] and White [21]. ${ }^{2}$ (See Section 2 for definitions and background material.) On the one hand, this result is a corollary to Theorem 1.1. On the other hand, Theorem 1.1 is the special case $C=R$, so our result generalises this one. This result is proved in Proofs 2.8 and 2.13, which are similar to the proof of [14, Theorem 4.2].

THEOREM 1.2. Let $M$ be a non-zero finitely generated $R$-module, and let $C$ be a semidualizing $R$-module. Then

(1) If $\mathrm{G}_{C}-\operatorname{dim}_{R}(M)=r<\infty$ and $M$ is in the Bass class $\mathcal{B}_{C}(R)$, then there exist an $R$-regular sequence $\underline{z}=z_{1}, \ldots, z_{r}$, integers $n_{0}, n_{1}, \ldots, n_{r} \geqslant 0$ with $n_{r} \geqslant 1$, and an exact sequence

$$
0 \rightarrow M \rightarrow Z \rightarrow N \rightarrow 0
$$

with $Z=\oplus_{i=0}^{r}\left(C /\left(\underline{z}_{[i]}\right) C\right)^{n_{i}}$ and $\mathrm{G}_{C}-\operatorname{dim}_{R}(N) \leqslant r$ and $N \in \mathcal{B}_{C}(R)$.

(2) If $\mathcal{P}_{C^{-}} \operatorname{pd}_{R}(M)=r<\infty$, then there exist an $R$-regular sequence $\underline{z}=z_{1}, \ldots, z_{r}$, integers $n_{0}, n_{1}, \ldots, n_{r} \geqslant 0$ with $n_{r} \geqslant 1$, and an exact sequence

$$
0 \rightarrow M \rightarrow Z \rightarrow N \rightarrow 0
$$

with $Z=\oplus_{i=0}^{r}\left(C /\left(\underline{z}_{[i]}\right) C\right)^{n_{i}}$ and $\mathcal{P}_{C}-\operatorname{pd}_{R}(N) \leqslant r$.

The next result we extend is the following:

TheOREM 1.3 [14, Theorem 4.2]. Let $R$ be a Cohen-Macaulay ring with a pointwise dualizing module $\omega$. Then, for any non-zero finitely generated $R$-module $M$ with locally finite injective dimension, there exist an integer $r=\operatorname{pd}_{R}\left(\operatorname{Hom}_{R}(\omega, M)\right)$, an R-regular sequence $\underline{z}=z_{1}, \ldots, z_{r}$, non-negative integers $n_{0}, n_{1}, \ldots, n_{r}$ with $n_{r} \geqslant 1$ and an exact sequence

$$
0 \rightarrow M \rightarrow Z \rightarrow N \rightarrow 0
$$

with $\left.Z=\oplus_{i=0}^{r}\left(\omega / \underline{z}_{[i]}\right) \omega\right)^{n_{i}}$ such that $N$ has locally finite injective dimension and $\operatorname{pd}_{R}\left(\operatorname{Hom}_{R}(\omega, N)\right) \leqslant r$.

We extend this in two directions in Theorems 1.4 and 1.5. First, we have the version for $\mathrm{G}$-injective dimension. Second, we give versions relative to a semidualizing module. These are proved in Proofs 3.5, 3.12 and 3.16.

THEOREM 1.4. Let R be a Cohen-Macaulay ring with a pointwise dualizing module $\omega$. For any non-zero finitely generated $R$-module $M$ with locally finite $G$-injective dimension, there exist an integer $r=\mathrm{G}-\operatorname{dim}_{R}\left(\operatorname{Hom}_{R}(\omega, M)\right)$, an R-regular sequence $\underline{z}=z_{1}, \ldots, z_{r}$, non-negative integers $n_{0}, n_{1}, \ldots, n_{r}$ with $n_{r} \geqslant 1$ and an exact sequence

$$
0 \rightarrow M \rightarrow Z \rightarrow N \rightarrow 0
$$

\footnotetext{
${ }^{2}$ It is worth noting that these notions appeared (more or less) implicitly in several places. However, to the best of our knowledge, these are the first places where $\mathrm{G}_{C}$-dimension and $\mathcal{P}_{C}$-projective dimension were studied explicitly.
} 
with $Z=\oplus_{i=0}^{r}\left(\omega /\left(\underline{z}_{[i]}\right) \omega\right)^{n_{i}}$ such that $N$ has locally finite $G$-injective dimension and $\mathrm{G}-\operatorname{dim}_{R}\left(\operatorname{Hom}_{R}(\omega, N)\right) \leqslant r$.

THEOREM 1.5. Let $R$ be a Cohen-Macaulay ring with a pointwise dualizing module $\omega$. Let $M$ a non-zero finitely generated $R$-module, and let $C$ be a semidualizing $R$-module. Set $C^{\dagger}=\operatorname{Hom}_{R}(C, \omega)$. Then

(1) If $M$ has locally finite $\mathcal{G I}_{C^{-}}$id and $M$ is in the Auslander class $\mathcal{A}_{C}(R)$, then there exist an integer $r=\mathrm{G}-\operatorname{dim}\left(\operatorname{Hom}_{R}\left(\omega, C \otimes_{R} M\right)\right)$, an $R$-regular sequence $\underline{z}=$ $z_{1}, \ldots, z_{r}$, non-negative integers $n_{0}, n_{1}, \ldots, n_{r}$ with $n_{r} \geqslant 1$ and an exact sequence

$$
0 \rightarrow M \rightarrow Z \rightarrow N \rightarrow 0
$$

such that $N$ has locally finite $\mathcal{G} \mathcal{I}_{C}$-id, and $Z=\oplus_{i=0}^{r}\left(C^{\dagger} /\left(\underline{z}_{[i]}\right) C^{\dagger}\right)^{n_{i}}, N \in \mathcal{A}_{C}(R)$ and $\mathrm{G}-\operatorname{dim}\left(\operatorname{Hom}_{R}\left(\omega, C \otimes_{R} N\right)\right) \leqslant r$.

(2) If $M$ has locally finite $\mathcal{I}_{C}$-id, then there exist an integer

$$
r=\operatorname{pd}_{R}\left(\operatorname{Hom}_{R}\left(\omega, C \otimes_{R} M\right)\right),
$$

an $R$-regular sequence $\underline{z}=z_{1}, \ldots, z_{r}$, non-negative integers $n_{0}, n_{1}, \ldots, n_{r}$ with $n_{r} \geqslant 1$ and an exact sequence

$$
0 \rightarrow M \rightarrow Z \rightarrow N \rightarrow 0
$$

such that $N$ has locally finite $\mathcal{I}_{C^{-}} \mathrm{id}$, and $Z=\oplus_{i=0}^{r}\left(C^{\dagger} /\left(\underline{z}_{[i]}\right) C^{\dagger}\right)^{n_{i}}$ and $\operatorname{pd}_{R}\left(\operatorname{Hom}_{R}\left(\omega, C \otimes_{R} N\right)\right) \leqslant r$.

We summarise the organisation of this paper. Section 2 contains the proof of Theorem 1.2, along with the necessary background material. Section 3 similarly treats Theorems 1.4 and 1.5; this section also contains several lemmas about modules of locally finite $\mathrm{G}$-injective dimension, locally finite $\mathrm{G}_{C}$-injective dimension and locally finite $\mathcal{I}_{C}$-injective dimension where $C$ is a semidualizing module, as these notions have not been developed in the literature as best we know. ${ }^{3}$ Note that we do not develop the background material in the most optimal manner, focusing instead on accessibility. To be specific, we present background material as it is needed in Sections 2 and 3 instead of putting it all in its own section. Also, we feel that the definition of locally finite $\mathcal{G I}_{C^{-}}$id is a bit much to swallow on the first bite, so we first discuss the special case of $\mathcal{G}$-id and work our way up.

\section{Proof of Theorem 1.2.}

NotATION 2.1. We let $\mathrm{pd}_{R}(-)$ and $\mathrm{id}_{R}(-)$ denote the classical projective dimension and the classical injective dimension, respectively.

The study of semidualizing modules was initiated independently (with different names) by Foxby [9], Golod [12] and Vasconcelos [20]. For example, a finitely generated projective $R$-module of rank 1 is semidualizing.

Definition 2.2. A finitely generated $R$-module $C$ is semidualizing if the natural map $R \rightarrow \operatorname{Hom}_{R}(C, C)$ is an isomorphism, and $\operatorname{Ext}_{R}^{\geqslant 1}(C, C)=0$. An $R$-module $C$ is

\footnotetext{
${ }^{3}$ This can be partially explained by the fact that at the time of the writing of this paper, the localisation question for G-injective dimension is still not completely answered. See Remark 3.2.
} 
pointwise dualizing if it is semidualizing and $\operatorname{id}_{R_{\mathfrak{m}}}\left(C_{\mathfrak{m}}\right)<\infty$ for all maximal ideals $\mathfrak{m} \subset R .^{4}$ An $R$-module $C$ is dualizing if it is semidualizing and $\operatorname{id}_{R}(C)<\infty$.

FACT 2.3.

(a) It is straightforward to show that the semidualizing property is local. That is, if $C$ is a semidualizing $R$-module, then for each multiplicatively closed subset $U \subseteq R$, the localisation $U^{-1} C$ is a semidualizing $U^{-1} R$-module; and conversely, if $M$ is a finitely generated $R$-module such that $M_{\mathfrak{m}}$ is a semidualizing $R_{\mathfrak{m}}$-module for each maximal ideal $\mathfrak{m} \subset R$, then $M$ is a semidualizing $R$-module. Similarly, if $C$ is a (pointwise) dualizing module for $R$, then for each multiplicatively closed subset $U \subseteq R$, the localisation $U^{-1} C$ is a (pointwise) dualizing module for $U^{-1} R$; and conversely, if $M$ is a finitely generated $R$-module such that $M_{\mathfrak{m}}$ is a dualizing module for $R_{\mathfrak{m}}$ for each maximal ideal $\mathfrak{m} \subset R$, then $M$ is a pointwise dualizing module for $R$. Moreover, an $R$-module $M$ is a dualizing module for $R$ if and only if it is a pointwise dualizing module for $R$ and the Krull dimension of $R$ is finite.

(b) Let $C$ be a semidualizing $R$-module. The isomorphism $R \cong \operatorname{Hom}_{R}(C, C)$ implies that $\operatorname{Supp}_{R}(C)=\operatorname{Spec}(R)$ and $\operatorname{Ass}_{R}(C)=\operatorname{Ass}(R)$. Thus, an element $x \in R$ is $C$-regular if and only if it is $R$-regular. When $x$ is $R$-regular, the quotient $C / x C$ is a semidualizing $R / x R$-module. Thus, by induction, a sequence $\underline{z}=z_{1}, \ldots, z_{r} \in R$ is $R$ regular if and only if it is $C$-regular; see [11, Theorem 4.5]. Also, from [16, Proposition 3.1], we know that for all $R$-modules $M \neq 0$, we have $C \otimes_{R} M \neq 0 \neq \operatorname{Hom}_{R}(C, M)$.

The next categories were introduced by Foxby [10] when $C$ is dualizing, and by Vasconcelos [20, Section 4.4] for arbitrary $C$, with different notation.

Definition 2.4. Let $C$ be a semidualizing $R$-module. The Auslander class of $C$ is the class $\mathcal{A}_{C}(R)$ of $R$-modules $M$ such that $\operatorname{Tor}_{\geqslant 1}^{R}(C, M)=0=\operatorname{Ext}_{R}^{\geqslant 1}\left(C, C \otimes_{R} M\right)$, and the natural map $M \rightarrow \operatorname{Hom}_{R}\left(C, C \otimes_{R} M\right)$ is an isomorphism. The Bass class of $C$ is the class $\mathcal{B}_{C}(R)$ of $R$-modules $N$ such that $\operatorname{Ext}_{R}^{\geqslant 1}(C, M)=0=\operatorname{Tor}_{\geqslant 1}^{R}\left(C, \operatorname{Hom}_{R}(C, M)\right)$, and the natural evaluation map $C \otimes_{R} \operatorname{Hom}_{R}(C, N) \rightarrow N$ is an isomorphism.

REMARK 2.5. It is straightforward to show that the Auslander and Bass classes for $C=R$ are trivial: $\mathcal{A}_{R}(R)$ and $\mathcal{B}_{R}(R)$ both contain all $R$-modules.

The following notion was introduced and studied by Holm and Jørgensen [15] and White [21]. Note that in the special case $C=R$, we recover the class of projective $R$-modules and the classical projective dimension.

Definition 2.6. Let $C$ be a semidualizing $R$-module. Let $\mathcal{P}_{C}(R)$ denote the class of modules $M \cong P \otimes_{R} C$ with $P$ projective. Modules in $\mathcal{P}_{C}(R)$ are called $C$-projective. We let $\mathcal{P}_{C}-\mathrm{pd}_{R}(-)$ denote the homological dimension obtained from resolutions in $\mathcal{P}_{C}(R)$, with the convention that $\mathcal{P}_{C^{-}} \operatorname{pd}_{R}(0)=-\infty .^{5}$

FACT 2.7. Let $C$ be a semidualizing $R$-module. The Bass class $\mathcal{B}_{C}(R)$ contains all $R$-modules of finite $\mathcal{P}_{C^{-}}$pd; see [16, Lemmas 4.1 and 5.1 and Corollary 6.3]. Given an $R$-module $M$, one has $\mathcal{P}_{C^{-}} \operatorname{pd}_{R}(M)=\operatorname{pd}_{R}\left(\operatorname{Hom}_{R}(C, M)\right)$ and $\operatorname{pd}_{R}(M)=\mathcal{P}_{C^{-}} \operatorname{pd}_{R}$ $\left(C \otimes_{R} M\right)$ by [19, Theorem 2.11]. In particular, one has $\mathcal{P}_{C^{-}} \operatorname{pd}_{R}(M)<\infty$ if and only if

\footnotetext{
$\overline{4}$ In [14], the term 'global canonical module' is used in place of 'pointwise dualizing module'. Our choice follows the terminology of Grothendieck and Hartshorne [13].

${ }^{5}$ We observe the same convention for any homological dimension of the zero module.
} 
$\operatorname{pd}_{R}\left(\operatorname{Hom}_{R}(C, M)\right)<\infty$, and one has $\operatorname{pd}_{R}(M)<\infty$ if and only if $\mathcal{P}_{C^{-}} \operatorname{pd}_{R}\left(C \otimes_{R} M\right)<$ $\infty$.

Proof 2.8 (Proof of Theorem 1.2(2)). Assume that $M$ is a non-zero finitely generated $R$-module such that $\mathcal{P}_{C}-\mathrm{pd}_{R}(M)=r<\infty$. Then Fact 2.7 implies that $\operatorname{pd}_{R}\left(\operatorname{Hom}_{R}(C, M)\right)=r<\infty$. Since $M \neq 0$, we have $\operatorname{Hom}_{R}(C, M) \neq 0$, by Fact 2.3(b), so Theorem 1.1(2) provides an $R$-regular sequence $\underline{z}=z_{1}, \ldots, z_{r}$, integers $n_{0}, n_{1}, \ldots, n_{r} \geqslant 0$ with $n_{r} \geqslant 1$, and an exact sequence

$$
0 \rightarrow \operatorname{Hom}_{R}(C, M) \rightarrow Z^{\prime} \rightarrow N^{\prime} \rightarrow 0,
$$

with $Z^{\prime}=\oplus_{i=0}^{r}\left(R /\left(\underline{z}_{[i]}\right)\right)^{n_{i}}$ and $\mathrm{pd}_{R}\left(N^{\prime}\right) \leqslant r$. In particular, we have $N^{\prime} \in \mathcal{A}_{C}(R)$ which implies that $\operatorname{Tor}_{1}^{R}\left(C, N^{\prime}\right)=0$. Thus, an application of $C \otimes_{R}-$ to the sequence (2.8.1) yields the next exact sequence:

$$
0 \rightarrow C \otimes_{R} \operatorname{Hom}_{R}(C, M) \rightarrow C \otimes_{R} Z^{\prime} \rightarrow C \otimes_{R} N^{\prime} \rightarrow 0 .
$$

Fact 2.7 implies that $M \in \mathcal{B}_{C}(R)$, so we have $C \otimes_{R} \operatorname{Hom}_{R}(C, M) \cong M$. The equality $Z^{\prime}=\oplus_{i=0}^{r}\left(R /\left(z_{[i]}\right)\right)^{n_{i}}$ implies that $Z:=C \otimes_{R} Z^{\prime}=\oplus_{i=0}^{r}\left(C /\left(z_{[i]}\right) C\right)^{n_{i}}$. Because of Fact 2.7, the condition $\operatorname{pd}_{R}\left(N^{\prime}\right) \leqslant r$ implies that $\mathcal{P}_{C^{-}} \operatorname{pd}_{R}\left(C \otimes_{R} N^{\prime}\right) \leqslant r$. Thus, with $N:=C \otimes_{R} N^{\prime}$, the sequence (2.8.2) satisfies the conclusion of Theorem 1.2(2).

The remainder of the proof of Theorem 1.2 is similar to the above proof, but it requires a bit more technology.

FACT 2.9. Let $C$ be a semidualizing $R$-module.

(a) The Auslander class $\mathcal{A}_{C}(R)$ contains all $R$-modules of finite projective dimension, and the Bass class $\mathcal{B}_{C}(R)$ contains all $R$-modules of finite injective dimension; see [16, Lemmas 4.1 and 5.1 and Corollary 6.3]. Moreover, it is straightforward to show that the Bass class satisfies the following local-global principal: For an $R$-module $M$, the following conditions are equivalent:

(i) $M \in \mathcal{B}_{C}(R)$;

(ii) $U^{-1} M \in \mathcal{B}_{U^{-1} C}\left(U^{-1} R\right)$ for each multiplicatively closed subset $U \subseteq R$;

(iii) $M_{\mathfrak{p}} \in \mathcal{B}_{C_{\mathfrak{p}}}\left(R_{\mathfrak{p}}\right)$ for each $\mathfrak{p} \in \operatorname{Spec}(R)$; and

(iv) $M_{\mathfrak{m}} \in \mathcal{B}_{C_{\mathfrak{m}}}\left(R_{\mathfrak{m}}\right)$ for each maximal ideal $\mathfrak{m} \in \operatorname{Supp}_{R}(M)$.

It follows that $\mathcal{B}_{C}(R)$ contains every $R$-module of locally finite injective dimension. The Auslander class satisfies an analogous local-global principal.

(b) The Auslander and Bass classes also satisfy the two-of-three property by [16, Corollary 6.3]. That is, given a short exact sequence $0 \rightarrow M^{\prime} \rightarrow M \rightarrow M^{\prime \prime} \rightarrow 0$ of $R$-module homomorphisms, if two of the modules in the sequence are in $\mathcal{A}_{C}(R)$, then so is the third module, and similarly for $\mathcal{B}_{C}(R)$.

(c) From [19, Theorem 2.8], we know that an $R$-module $M$ is in $\mathcal{B}_{C}(R)$ if and only if $\operatorname{Hom}_{R}(C, M) \in \mathcal{A}_{C}(R)$, and that $M \in \mathcal{A}_{C}(R)$ if and only if $C \otimes_{R} M \in \mathcal{B}_{C}(R)$. This is known as Foxby equivalence.

Definition 2.10. Let $C$ be a semidualizing $R$-module. A finitely generated $R$ module $G$ is totally $C$-reflexive if the natural map $G \rightarrow \operatorname{Hom}_{R}\left(\operatorname{Hom}_{R}(G, C), C\right)$ is an isomorphism, and $\operatorname{Ext}_{R}^{\geqslant 1}(G, C)=0=\operatorname{Ext}_{R}^{\geqslant 1}\left(\operatorname{Hom}_{R}(G, C), C\right)$. Let $\mathcal{G}_{C}(R)$ denote the class of totally $C$-reflexive $R$-modules, and set $\mathcal{G}(C)=\mathcal{G}_{C}(R) \cap \mathcal{B}_{C}(R)$. In the case $C=R$, we use the more common terminology 'totally reflexive' and the notation 
$\mathcal{G}(R)=\mathcal{G}_{R}(R)=\mathcal{G}(R) \cap \mathcal{B}_{R}(R)$. We abbreviate as follows:

$\mathrm{G}_{C}-\operatorname{dim}_{R}(-)=$ the homological dimension obtained from resolutions in $\mathcal{G}_{C}(R)$,

$\mathcal{G}(C)-\operatorname{pd}_{R}(-)=$ the homological dimension obtained from resolutions in $\mathcal{G}(C)$,

G- $\operatorname{dim}_{R}(-)=$ the homological dimension obtained from resolutions in $\mathcal{G}(R)$.

The following facts are included for perspective.

FACT 2.11. Let $C$ be a semidualizing $R$-module, and let $M$ be a finitely generated $R$ module. Because of the containments $\mathcal{P}_{C}(R) \subseteq \mathcal{G}(C) \subseteq \mathcal{G}_{C}(R)$, one has $\mathrm{G}_{C}$-dim $\operatorname{dim}_{R}(M) \leqslant$ $\mathcal{G}(C)-\mathrm{pd}_{R}(M) \leqslant \mathcal{P}_{C^{-}}-\mathrm{pd}_{R}(M)$ with equality to the left of any finite quantity. In particular, the case $C=R$ says that $\mathrm{G}-\operatorname{dim}_{R}(M)=\mathcal{G}(R)-\operatorname{pd}_{R}(M) \leqslant \operatorname{pd}_{R}(M)$ since $\mathcal{G}(R)=\mathcal{G}_{R}(R)$, with equality holding when $\operatorname{pd}_{R}(M)<\infty$.

The next lemma explains how these homological dimensions are connected.

Lemma 2.12 [18, Lemma 2.9]. Let $C$ be a semidualizing $R$-module. For a finitely generated $R$-module $M$, the following conditions are equivalent:

(i) $\mathcal{G}(C)-\operatorname{pd}_{R}(M)<\infty$;

(ii) $\mathrm{G}_{C}-\operatorname{dim}_{R}(M)<\infty$ and $M \in \mathcal{B}_{C}(R)$; and

(iii) $\mathrm{G}-\operatorname{dim}_{R}\left(\operatorname{Hom}_{R}(C, M)\right)<\infty$ and $M \in \mathcal{B}_{C}(R)$.

When these conditions are satisfied, we have

$$
\mathcal{G}(C)-\operatorname{pd}_{R}(M)=\mathrm{G}_{C}-\operatorname{dim}_{R}(M)=\mathrm{G}-\operatorname{dim}_{R}\left(\operatorname{Hom}_{R}(C, M)\right) .
$$

Now we are in position to complete the proof of Theorem 1.2.

Proof 2.13 (Proof of Theorem 1.2(1)). Assume that $M$ is a non-zero finitely generated $R$-module such that $\mathrm{G}_{C}-\operatorname{dim}_{R}(M)=r<\infty$ and $M \in \mathcal{B}_{C}(R)$. Then Lemma 2.12 implies that $\mathrm{G}-\operatorname{dim}_{R}\left(\operatorname{Hom}_{R}(C, M)\right)=r<\infty$. Since $M \neq 0$, we have $\operatorname{Hom}_{R}(C, M) \neq 0$, by Fact 2.3(b), so Theorem 1.1(1) provides an $R$-regular sequence $\underline{z}=z_{1}, \ldots, z_{r}$, integers $n_{0}, n_{1}, \ldots, n_{r} \geqslant 0$ such that $n \geqslant 1$, and an exact sequence

$$
0 \rightarrow \operatorname{Hom}_{R}(C, M) \rightarrow Z^{\prime} \rightarrow N^{\prime} \rightarrow 0,
$$

with $Z^{\prime}=\oplus_{i=0}^{r}\left(R /\left(\underline{z}_{[i]}\right)\right)^{n_{i}}$ and G-dim ${ }_{R}\left(N^{\prime}\right) \leqslant r$.

The condition $M \in \mathcal{B}_{C}(R)$ implies that $\operatorname{Hom}_{R}(C, M) \in \mathcal{A}_{C}(R)$ by Fact 2.9(c), and Fact 2.9(a) implies that $Z^{\prime} \in \mathcal{A}_{C}(R)$. Also, from Fact 2.9(a)-(b), we conclude that $N^{\prime} \in \mathcal{A}_{C}(R)$, which implies that $\operatorname{Tor}_{1}^{R}\left(C, N^{\prime}\right)=0$. Thus, an application of $C \otimes_{R}-$ to the sequence (2.13.1) yields the next exact sequence:

$$
0 \rightarrow C \otimes_{R} \operatorname{Hom}_{R}(C, M) \rightarrow C \otimes_{R} Z^{\prime} \rightarrow C \otimes_{R} N^{\prime} \rightarrow 0 .
$$

The assumption $M \in \mathcal{B}_{C}(R)$ gives an isomorphism $C \otimes_{R} \operatorname{Hom}_{R}(C, M) \cong M$. The equality $Z^{\prime}=\oplus_{i=0}^{r}\left(R /\left(\underline{z}_{[i]}\right)\right)^{n_{i}}$ implies that $Z:=C \otimes_{R} Z^{\prime}=\oplus_{i=0}^{r}\left(C /\left(\underline{z}_{[i]}\right) C\right)^{n_{i}}$.

The condition $N^{\prime} \in \mathcal{A}_{C}(R)$ implies that $N:=C \otimes_{R} N^{\prime} \in \mathcal{B}_{C}(R)$ by Fact 2.9(c), and $N^{\prime} \cong \operatorname{Hom}_{R}(C, N)$ by the definition of what it means for $N^{\prime}$ to be in $\mathcal{A}_{C}(R)$. Thus, because of Lemma 2.12, we have

$$
\mathrm{G}_{C}-\operatorname{dim}_{R}(N)=\mathrm{G}-\operatorname{dim}\left(\operatorname{Hom}_{R}(C, N)\right)=\mathrm{G}-\operatorname{dim}_{R}\left(N^{\prime}\right) \leqslant r .
$$

Thus, the sequence (2.13.2) satisfies the conclusion of Theorem 1.2(1). 
3. Proofs of Theorems 1.4 and 1.5. We continue with a definition due to Enochs and Jenda [7].

Definition 3.1. A complete injective resolution is an exact complex $Y$ of injective $R$-modules such that $\operatorname{Hom}_{R}(J, Y)$ is exact for each injective $R$-module $J$. An $R$ module $N$ is $G$-injective if there exists a complete injective resolution $Y$ such that $N \cong \operatorname{Ker}\left(\partial_{0}^{Y}\right)$, and $Y$ is a complete injective resolution of $N$. Let $\mathcal{G I}(R)$ denote the class of G-injective $R$-modules, and let $\operatorname{Gid}_{R}(-)$ denote the homological dimension obtained from co-resolutions in $\mathcal{G} \mathcal{I}(R)$. We say that an $R$-module $M$ has locally finite $G$-injective dimension, provided that $\operatorname{Gid}_{R_{\mathfrak{m}}}\left(M_{\mathfrak{m}}\right)<\infty$ for each maximal ideal $\mathfrak{m} \subset R$.

REMARK 3.2. Using existing technology, the modules of finite G-injective dimension behave best when $R$ is Cohen-Macaulay with a dualizing module. ${ }^{6}$ For instance, in general, it is not known whether the G-injective dimension localises; but it is known to localise when $R$ has a dualizing module. This is due to the following connection with Bass classes, the local case of which is from $[8]{ }^{7}$

Lemma 3.3. Assume that $R$ is Cohen-Macaulay with a pointwise dualizing module. Let $M$ be an R-module. Then an R-module $M$ has locally finite $G$-injective dimension if and only if $M \in \mathcal{B}_{\omega}(R)$.

Proof. If $R$ is local, then the result follows from [8, Proposition 1.4 and Theorems 1.6 and 2.5]. By definition, the condition ' $M$ has locally finite G-injective dimension' is a local condition. Fact 2.9(b) shows that the condition ' $M \in \mathcal{B}_{\omega}(R)$ ' is also a local condition. Note that Fact 2.3(a) implies that for each maximal ideal $\mathfrak{m} \subset R$, the localisation $\omega_{\mathfrak{m}}$ is a dualizing module for the Cohen-Macaulay local ring $R_{\mathfrak{m}}$. Thus, the general result follows from the local case.

The next result shows that the G-dimension of a finitely generated module behaves like its projective dimension.

Lemma 3.4. Let $M$ be a finitely generated R-module. The following conditions are equivalent:

(i) $\mathrm{G}-\operatorname{dim}_{R}(M)<\infty$;

(ii) $\mathrm{G}-\operatorname{dim}_{R_{\mathfrak{m}}}\left(M_{\mathfrak{m}}\right)<\infty$ for each maximal ideal $\mathfrak{m} \subset R$.

When $R$ is Cohen-Macaulay with a pointwise dualizing module $\omega$, these conditions are equivalent to the following:

(iii) $M \in \mathcal{A}_{\omega}(R)$.

Proof. The implication (i) $\Longrightarrow$ (ii) follows from the inequality G-dim $R_{\mathfrak{m}}\left(M_{\mathfrak{m}}\right) \leqslant$ G- $\operatorname{dim}_{R}(M)$, which is straightforward to verify. The implication (ii) $\Longrightarrow$ (i) is from [4, Theorem 3.3]. When $R$ is Cohen-Macaulay with a pointwise dualizing module $\omega$, the equivalence (ii) $\Longleftrightarrow$ (iii) follows from the local results [8, Proposition 1.3 and Theorems 1.6 and 2.1] as in the proof of Lemma 3.3. ${ }^{8}$

\footnotetext{
${ }^{6}$ Most good behaviour is also known when $R$ has a dualizing complex, but we restrict our attention to the Cohen-Macaulay case. For instance, the conclusion of Lemma 3.3 holds when $R$ is only assumed to have a pointwise dualizing complex; the proof is the same, using results from [6] in place of the results from [8].

${ }^{7}$ It is worth noting that the results in [8] assume that $R$ is local, hence with finite Krull dimension; also, the results of [6] assume implicitly that $R$ has finite Krull dimension since the definition of a dualizing complex used there includes an assumption of finite injective dimension. Contrast this with our definition of pointwise dualizing module, and with Grothendieck's definition of a pointwise dualizing complex from [13]. ${ }^{8}$ Note that this uses the characterisation of totally reflexive modules in terms of 'complete resolutions' found in [3, (4.4.4)]; see also [5, Theorem 3.1]. Specifically, an $R$-module $M$ is totally reflexive if and only if there
} 
Proof 3.5 (Proof of Theorem 1.4). Assume that $R$ is Cohen-Macaulay with a pointwise dualizing module $\omega$ and $M$ is a non-zero finitely generated $R$-module with locally finite G-injective dimension. Lemma 3.3 implies that $M \in \mathcal{B}_{\omega}(R)$. Using Foxby equivalence (from Fact 2.9(c)), we conclude that $\operatorname{Hom}_{R}(\omega, M) \in \mathcal{A}_{\omega}(R)$, so Lemma 3.4 implies that $r:=\mathrm{G}-\operatorname{dim}_{R}\left(\operatorname{Hom}_{R}(\omega, M)\right)<\infty$. The fact that $R$ is Noetherian implies that $\operatorname{Hom}_{R}(\omega, M)$ is finitely generated. (The proof concludes as in Proof 2.13. We include the details for the sake of thoroughness.)

Since $M \neq 0$, we have $\operatorname{Hom}_{R}(\omega, M) \neq 0$, by Fact 2.3(b), so Theorem 1.1(1) implies that there are an $R$-regular sequence $\underline{z}=z_{1}, \ldots, z_{r}$, integers $n_{0}, n_{1}, \ldots, n_{r} \geqslant 0$ with $n_{r} \geqslant 1$, and an exact sequence

$$
0 \rightarrow \operatorname{Hom}_{R}(\omega, M) \rightarrow Z^{\prime} \rightarrow N^{\prime} \rightarrow 0,
$$

with $Z^{\prime}=\oplus_{i=0}^{r}\left(R /\left(\underline{z}_{[i]}\right)\right)^{n_{i}}$ and G-dim${ }_{R}\left(N^{\prime}\right) \leqslant r$. Fact 2.9(a) implies that $Z^{\prime} \in \mathcal{A}_{\omega}(R)$. From Fact 2.9(b), we conclude that $N^{\prime} \in \mathcal{A}_{\omega}(R)$, which implies that $\operatorname{Tor}_{1}^{R}\left(\omega, N^{\prime}\right)=0$. Thus, an application of $\omega \otimes_{R}-$ to the sequence (3.5.1) yields the next exact sequence:

$$
0 \rightarrow \omega \otimes_{R} \operatorname{Hom}_{R}(\omega, M) \rightarrow \omega \otimes_{R} Z^{\prime} \rightarrow \omega \otimes_{R} N^{\prime} \rightarrow 0 .
$$

The assumption $M \in \mathcal{B}_{\omega}(R)$ gives an isomorphism $\omega \otimes_{R} \operatorname{Hom}_{R}(\omega, M) \cong M$. The equality $Z^{\prime}=\oplus_{i=0}^{r}\left(R /\left(\underline{z}_{[i]}\right)\right)^{n_{i}}$ implies that $Z:=\omega \otimes_{R} Z^{\prime}=\oplus_{i=0}^{r}\left(\omega /\left(\underline{z}_{[i]}\right) \omega\right)^{n_{i}}$.

The condition $N^{\prime} \in \mathcal{A}_{\omega}(R)$ implies that $N:=\omega \otimes_{R} N^{\prime} \in \mathcal{B}_{\omega}(R)$ by Fact 2.9(c), and $N^{\prime} \cong \operatorname{Hom}_{R}(\omega, N)$ by the definition of what it means for $N^{\prime}$ to be in $\mathcal{A}_{\omega}(R)$. Thus, we have G-dim $\left(\operatorname{Hom}_{R}(\omega, N)\right)=\mathrm{G}-\operatorname{dim}_{R}\left(N^{\prime}\right) \leqslant r$. So, the sequence (3.5.2) satisfies the conclusion of Theorem 1.4.

For our next results, we need a better understanding of the relationship between semidualizing modules and a pointwise dualizing module.

Lemma 3.6. Assume that $R$ is Cohen-Macaulay with a pointwise dualizing module $\omega$. If $C$ is a semidualizing $R$-module, then so is $\operatorname{Hom}_{R}(C, \omega)$.

Proof. In the local case, this result is standard; see, e.g. [17, Facts 1.18-1.19]. Since the semidualizing and (pointwise) dualizing conditions are local by Fact 2.3(a), the general case of the result follows.

The next two lemmas elaborate on the local-global behavior for our invariants.

Lemma 3.7. Let $M$ be a finitely generated $R$-module, and let $C$ be a semidualizing $R$-module. Then $\mathcal{P}_{C^{-}} \mathrm{pd}_{R}(M)<\infty$ if and only if $P_{C_{\mathfrak{m}}}-\operatorname{pd}_{R_{\mathfrak{m}}}\left(M_{\mathfrak{m}}\right)<\infty$ for each maximal ideal $\mathfrak{m} \subset R$.

Proof. The forward implication follows from the inequality $\mathrm{P}_{C_{\mathfrak{m}}}-\operatorname{dim}_{R_{\mathfrak{m}}}\left(M_{\mathfrak{m}}\right) \leqslant$ $\mathcal{P}_{C}-\operatorname{pd}_{R}(M)$, which is straightforward to verify. For the converse, assume that $\mathrm{P}_{C_{\mathfrak{m}}}-\operatorname{pd}_{R_{\mathfrak{m}}}\left(M_{\mathfrak{m}}\right)<\infty$ for each maximal ideal $\mathfrak{m} \subset R$. It follows that the module $\operatorname{Hom}_{R}(C, M)_{\mathfrak{m}} \cong \operatorname{Hom}_{R_{\mathfrak{m}}}\left(C_{\mathfrak{m}}, M_{\mathfrak{m}}\right)$ has finite projective dimension over $R_{\mathfrak{m}}$ for all $\mathfrak{m}$. Hence, the finitely generated $R$-module $\operatorname{Hom}_{R}(C, M)$ has finite projective dimension, so Fact 2.7 implies that $\mathcal{P}_{C^{-}} \operatorname{pd}_{R}(M)$ is finite.

is an exact complex $F=\cdots \stackrel{\partial_{1}^{F}}{\longrightarrow} F_{0} \stackrel{\partial_{0}^{F}}{\longrightarrow} F_{-1} \stackrel{\partial_{-1}^{F}}{\longrightarrow} \cdots$ such that $\operatorname{Hom}_{R}(F, R)$ is exact and $M \cong \operatorname{Im}\left(\partial_{0}^{F}\right)$. Note that the local result was also announced in [10, Corollary 3.3]. 
The next lemma is a souped-up version of a special case of a result of Takahashi and White that is documented in [18].

Lemma 3.8. Assume that $R$ is Cohen-Macaulay with a pointwise dualizing module $\omega$. For each finitely generated $R$-module $M$, one has $\mathcal{P}_{\omega}-\operatorname{pd}_{R}(M)<\infty$ if and only if $M$ has locally finite injective dimension.

Proof. When $\omega$ is a dualizing module for $R$, i.e. when $R$ has finite Krull dimension, this result is from [18, Lemma 2.7]. In particular, this takes care of the local case. The general case follows from the local case, by Lemma 3.7.

Here are some more notions from [15].

Definition 3.9. Let $C$ be a semidualizing $R$-module, and let $\mathcal{I}_{C}(R)$ denote the class of modules $N \cong \operatorname{Hom}_{R}(C, I)$ with $I$ injective. Modules in $\mathcal{I}_{C}(R)$ are called $C$ injective. We let $\mathcal{I}_{C}-\mathrm{id}_{R}(-)$ denote the homological dimension obtained from coresolutions in $\mathcal{I}_{C}(R)$. We say that an $R$-module $M$ has locally finite $\mathcal{I}_{C}$-id, provided that $\mathrm{I}_{C_{\mathfrak{m}}}-\operatorname{id}_{R_{\mathfrak{m}}}\left(M_{\mathfrak{m}}\right)<\infty$ for each maximal ideal $\mathfrak{m} \subset R$.

FACT 3.10. Let $C$ be a semidualizing $R$-module. The Auslander class $\mathcal{A}_{C}(R)$ contains every $R$-module of finite $\mathcal{I}_{C}$-injective dimension; see [16, Lemmas 4.1 and 5.1]. Given an $R$-module $M$, one has $\mathcal{I}_{C}-\mathrm{id}_{R}(M)=\operatorname{id}_{R}\left(C \otimes_{R} M\right)$ and $\operatorname{id}_{R}(M)=$ $\mathcal{I}_{C}-\mathrm{id}_{R}\left(\operatorname{Hom}_{R}(C, M)\right)$ by [19, Theorem 2.11]. In particular, one has $\mathcal{I}_{C}-\mathrm{id}_{R}(M)<$ $\infty$ if and only if $\operatorname{id}_{R}\left(C \otimes_{R} M\right)<\infty$, and one has $\operatorname{id}_{R}(M)<\infty$ if and only if $\mathcal{I}_{C}-\mathrm{id}_{R}\left(\operatorname{Hom}_{R}(C, M)\right)<\infty$. It follows that $M$ has locally finite $\mathcal{I}_{C}$-id if and only if $C \otimes_{R} M$ has locally finite injective dimension, and $M$ has locally finite injective dimension if and only if $\operatorname{Hom}_{R}(C, M)$ has locally finite $\mathcal{I}_{C}$-id.

Lemma 3.11. Assume that $R$ is Cohen-Macaulay with a pointwise dualizing module $\omega$. Let $C$ be a semidualizing $R$-module, and let $x=x_{1}, \ldots, x_{i} \in R$ be an $R$-regular sequence. Then $\operatorname{Hom}_{R}(C, \omega /(\underline{x}) \omega) \cong C^{\dagger} /(\underline{x}) C^{\dagger}$, where $C^{\dagger}=\operatorname{Hom}_{R}(C, \omega)$.

Proof. Let $K$ be the Koszul complex $K^{R}(\underline{x})$, and let $K^{+}$denote the augmented Koszul complex

$$
K^{+}=(0 \rightarrow R \rightarrow \cdots \rightarrow R \rightarrow \underbrace{R /(\underline{x})}_{\text {deg. }-1} \rightarrow 0) .
$$

Since $x$ is $R$-regular, $K^{+}$is an exact sequence of $R$-modules of finite projective dimension. Thus, each module $K_{j}^{+}$is in $\mathcal{A}_{\omega}(R)$, so the induced complex

$$
\omega \otimes_{R} K^{+}=(0 \rightarrow \omega \rightarrow \cdots \rightarrow \omega \rightarrow \underbrace{\omega /(\underline{x}) \omega}_{\text {deg. }-1} \rightarrow 0)
$$

is an exact sequence of $R$-modules locally of finite injective dimension. In particular, the modules in this exact sequence are in $\mathcal{B}_{C}(R)$, so the next sequence is also exact:

$$
\begin{aligned}
& \operatorname{Hom}_{R}\left(C, \omega \otimes_{R} K^{+}\right) \\
& \quad=(0 \rightarrow \operatorname{Hom}_{R}(C, \omega) \rightarrow \cdots \rightarrow \operatorname{Hom}_{R}(C, \omega) \rightarrow \underbrace{\operatorname{Hom}_{R}(C, \omega /(\underline{x}) \omega)}_{\text {deg. }-1} \rightarrow 0) .
\end{aligned}
$$


It is straightforward to show that the differential on this sequence in positive degrees is the same as the differential on $C^{\dagger} \otimes_{R} K$. It follows that

$$
\operatorname{Hom}_{R}(C, \omega /(\underline{x}) \omega) \cong \mathrm{H}_{0}\left(C^{\dagger} \otimes_{R} K\right) \cong C^{\dagger} /(\underline{x}) C^{\dagger},
$$

as desired.

Proof 3.12 (Proof of Theorem 1.5(2)). Assume that $R$ is Cohen-Macaulay with a pointwise dualizing module $\omega$ and $M$ is a non-zero finitely generated $R$-module with locally finite $\mathcal{I}_{C}$-id. Fact 3.10 implies that $C \otimes_{R} M$ has locally finite injective dimension. Since $M \neq 0$, we have $C \otimes_{R} M \neq 0$, by Fact 2.3(b), so Theorem 1.3 provides an integer $r=\operatorname{pd}_{R}\left(\operatorname{Hom}_{R}\left(\omega, C \otimes_{R} M\right)\right)$, a proper $R$-regular sequence $\underline{z}=z_{1}, \ldots, z_{r}$, non-negative integers $n_{0}, n_{1}, \ldots, n_{r}$ with $n_{r} \geqslant 1$, and an exact sequence

$$
0 \rightarrow C \otimes_{R} M \rightarrow Z^{\prime} \rightarrow N^{\prime} \rightarrow 0,
$$

with $Z^{\prime}=\oplus_{i=0}^{r}\left(\omega /\left(\underline{z}_{[i]}\right) \omega\right)^{n_{i}}$, where $N^{\prime}$ has locally finite injective dimension and $\operatorname{pd}_{R}\left(\operatorname{Hom}_{R}\left(\omega, N^{\prime}\right)\right) \leqslant r$.

As $C \otimes_{R} M$ has locally finite injective dimension, we have $C \otimes_{R} M \in \mathcal{B}_{C}(R)$ by Fact 2.9 (b), so $\operatorname{Ext}_{R}^{1}\left(C, C \otimes_{R} M\right)=0$ and $\operatorname{Hom}_{R}\left(C, C \otimes_{R} M\right) \cong M$. Thus, an application of $\operatorname{Hom}_{R}(C,-)$ to the sequence (3.12.1) yields the next exact sequence:

$$
0 \rightarrow M \rightarrow \operatorname{Hom}_{R}\left(C, Z^{\prime}\right) \rightarrow \operatorname{Hom}_{R}\left(C, N^{\prime}\right) \rightarrow 0 .
$$

Since $N^{\prime}$ has locally finite injective dimension, Fact 3.10 implies that the $R$-module $N:=\operatorname{Hom}_{R}\left(C, N^{\prime}\right)$ has locally finite $\mathcal{I}_{C}$-id. With $Z:=\operatorname{Hom}_{R}\left(C, Z^{\prime}\right)$, it remains to show that $Z \cong \oplus_{i=0}^{r}\left(C^{\dagger} /\left(\underline{z}_{[i]}\right) C^{\dagger}\right)^{n_{i}}$ and $\operatorname{pd}_{R}\left(\operatorname{Hom}_{R}\left(\omega, C \otimes_{R} N\right)\right) \leqslant r$. The first of these follows from the next sequence of isomorphisms

$$
\begin{aligned}
Z & \cong \operatorname{Hom}_{R}\left(C, \oplus_{i=0}^{r}\left(\omega /\left(\underline{z}_{[i]}\right) \omega\right)^{n_{i}}\right) \\
& \cong \oplus_{i=0}^{r} \operatorname{Hom}_{R}\left(C, \omega \otimes_{R}\left(R /\left(\underline{z}_{[i]}\right)\right)^{n_{i}}\right. \\
& \cong \oplus_{i=0}^{r}\left(C^{\dagger} /\left(\underline{z}_{[i]}\right) C^{\dagger}\right)^{n_{i}},
\end{aligned}
$$

where the last isomorphism is from Lemma 3.11. To complete the proof, observe that since $N^{\prime}$ has locally finite injective dimension, it is in $\mathcal{B}_{C}(R)$, so we have $N^{\prime} \cong C \otimes_{R} N$. This implies that $\operatorname{pd}_{R}\left(\operatorname{Hom}_{R}\left(\omega, C \otimes_{R} N\right)\right)=\operatorname{pd}_{R}\left(\operatorname{Hom}_{R}\left(\omega, N^{\prime}\right)\right) \leqslant r$, as desired.

In the next definition, the special case $C=R$ recovers the complete injective resolutions and Gorenstein injective modules.

Definition 3.13. Let $C$ be a semidualizing $R$-module. A complete $\mathcal{I}_{C} \mathcal{I}$-co-resolution is a complex $Y$ of $R$-modules such that $Y$ is exact and $\operatorname{Hom}_{R}(U, Y)$ is exact for each $U \in \mathcal{I}_{C}(R), Y_{i}$ is injective for $i \leqslant 0$ and $Y_{i}$ is $C$-injective for $i>0$. An $R$-module $N$ is $G_{C}$-injective if there exists a complete $\mathcal{I}_{C} \mathcal{I}$-co-resolution $Y$ such that $N \cong \operatorname{Ker}\left(\partial_{0}^{Y}\right)$, and $Y$ is a complete $\mathcal{I}_{C} \mathcal{I}$-co-resolution of $N$. Let $\mathcal{G I}_{C}(R)$ denote the class of $\mathrm{G}_{C}$-injective $R$-modules, and set $\mathcal{G}\left(\mathcal{I}_{C}\right)=\mathcal{G} \mathcal{I}_{C}(R) \cap \mathcal{A}_{C}(R)$. We let $\mathcal{G} \mathcal{I}_{C}-\mathrm{id}_{R}(-)$ and $\mathcal{G}\left(\mathcal{I}_{C}\right)-\mathrm{id}_{R}(-)$ denote the homological dimensions obtained from co-resolutions in $\mathcal{G} \mathcal{I}_{C}(R)$ and $\mathcal{G}\left(\mathcal{I}_{C}\right)$, respectively.

An $R$-module $M$ has locally finite $\mathcal{G}\left(\mathcal{I}_{C}\right)$-id, provided that $\mathcal{G}\left(\mathcal{I}_{C_{\mathfrak{m}}}\right)-\mathrm{id}_{R_{\mathfrak{m}}}\left(M_{\mathfrak{m}}\right)<\infty$ for each maximal ideal $\mathfrak{m} \subset R$. An $R$-module $M$ has locally finite $\mathcal{G I}_{C^{-}}$id, provided that $\mathcal{G I}_{C_{\mathfrak{m}}}-\mathrm{id}_{R_{\mathfrak{m}}}\left(M_{\mathfrak{m}}\right)<\infty$ for each maximal ideal $\mathfrak{m} \subset R$. 
The next lemma explains the relation between the different Gorenstein injective dimensions.

Lemma 3.14 [18, Lemma 2.10]. Let $C$ be a semidualizing $R$-module. For an $R$ module $M$, the following conditions are equivalent:

(i) $\mathcal{G}\left(\mathcal{I}_{C}\right)-\mathrm{id}_{R}(M)<\infty$;

(ii) $\mathcal{G I}_{C^{-}} \mathrm{id}_{R}(M)<\infty$ and $M \in \mathcal{A}_{C}(R)$; and

(iii) $\operatorname{Gid}_{R}\left(C \otimes_{R} M\right)<\infty$ and $M \in \mathcal{A}_{C}(R)$.

When these conditions are satisfied, we have

$$
\mathcal{G}\left(\mathcal{I}_{C}\right)-\mathrm{id}_{R}(M)=\mathcal{G} \mathcal{I}_{C^{-}} \mathrm{id}_{R}(M)=\operatorname{Gid}_{R}\left(C \otimes_{R} M\right) .
$$

We actually need the following corollary of the previous result.

Lemma 3.15. Let $C$ be a semidualizing $R$-module. For an $R$-module $M$, the following conditions are equivalent:

(i) $M$ has locally finite $\mathcal{G}\left(\mathcal{I}_{C}\right)$ - id;

(ii) $M$ has locally finite $\mathcal{G I}_{C}$-id and $M \in \mathcal{A}_{C}(R)$; and

(iii) $C \otimes_{R} M$ has locally finite Gid and $M \in \mathcal{A}_{C}(R)$.

Proof. This follows from the definitions of the locally finite Gorenstein injective dimensions and the local-global principal for Auslander classes from Fact 2.9(b).

Proof 3.16 (Proof of Theorem 1.5(1)). Assume that $R$ is Cohen-Macaulay with a pointwise dualizing module $\omega$ and $M$ is a non-zero finitely generated $R$-module in $\mathcal{A}_{C}(R)$ with locally finite $\mathcal{G I}_{C}$-id. Lemma 3.15 implies that $C \otimes_{R} M$ has locally finite G-injective dimension. Since $M \neq 0$, we have $C \otimes_{R} M \neq 0$, by Fact 2.3(b), so Theorem 1.4 provides an integer $r=\mathrm{G}$ - $\operatorname{dim}_{R}\left(\operatorname{Hom}_{R}\left(\omega, C \otimes_{R} M\right)\right)$, a proper $R$-regular sequence $\underline{z}=z_{1}, \ldots, z_{r}$, non-negative integers $n_{0}, n_{1}, \ldots, n_{r}$ with $n_{r} \geqslant 1$, and an exact sequence

$$
0 \rightarrow C \otimes_{R} M \rightarrow Z^{\prime} \rightarrow N^{\prime} \rightarrow 0
$$

such that $Z^{\prime}=\oplus_{i=0}^{r}\left(\omega /\left(\underline{z}_{[i]}\right) \omega\right)^{n_{i}}, N^{\prime} \in \mathcal{B}_{\omega}(R)$, and G-dim $\left.\operatorname{dim}_{R}\left(\omega, N^{\prime}\right)\right) \leqslant r$.

Since $M$ is in $\mathcal{A}_{C}(R)$, we have $\operatorname{Ext}_{R}^{1}\left(C, C \otimes_{R} M\right)=0$ and $\operatorname{Hom}_{R}\left(C, C \otimes_{R} M\right) \cong M$. Thus, an application of $\operatorname{Hom}_{R}(C,-)$ to the sequence $(3.16 .1)$ yields the next exact sequence:

$$
0 \rightarrow M \rightarrow \operatorname{Hom}_{R}\left(C, Z^{\prime}\right) \rightarrow \operatorname{Hom}_{R}\left(C, N^{\prime}\right) \rightarrow 0
$$

Note that $Z^{\prime}$ has locally finite injective dimension, so we have $Z^{\prime} \in \mathcal{B}_{C}(R)$, which implies that $\operatorname{Hom}_{R}\left(C, Z^{\prime}\right)$ and $\operatorname{Hom}_{R}\left(C, N^{\prime}\right)$ are in $\mathcal{A}_{C}(R)$ by Fact 2.9(b)-(c). With $Z:=$ $\operatorname{Hom}_{R}\left(C, Z^{\prime}\right)$, we have G-dim ${ }_{R}\left(\operatorname{Hom}_{R}\left(\omega, C \otimes_{R} N\right)\right) \leqslant r$ and $Z \cong \oplus_{i=0}^{r}\left(C^{\dagger} /\left(\underline{z}_{[i]}\right) C^{\dagger}\right)^{n_{i}}$, as in Proof 3.12.

ACKNOWLEDGEMENTS. The author is grateful to the referee for her/his thoughtful comments. This material is based on work supported by North Dakota EPSCoR and National Science Foundation Grant EPS-0814442. He was supported in part by a grant from the NSA. 


\section{REFERENCES}

1. M. Auslander, Anneaux de Gorenstein, et torsion en algèbre commutative, in Séminaire d'algèbre commutative dirigé par pierre samuel, vol. 1966/67 (Secrétariat mathématique, Paris, 1967), MR 37 \#1435.

2. M. Auslander and M. Bridger, Stable module theory, Memoirs of the American Mathematical Society, No. 94 (Amer. Math. Soc., Providence, RI, 1969), MR 42 \#4580.

3. L. L. Avramov and R.-O. Buchweitz, Support varieties and cohomology over complete intersections, Invent. Math. 142(2) (2000), 285-318. MR 1794064 (2001j:13017).

4. L. L. Avramov, S. B. Iyengar and J. Lipman, Reflexivity and rigidity for complexes. I. Commutative rings, Algebra Number Theory 4(1) (2010), 47-86. MR 2592013.

5. L. L. Avramov and A. Martsinkovsky, Absolute, relative, and Tate cohomology of modules of finite Gorenstein dimension, Proc. London Math. Soc. 85(3) (2002), 393-440. MR 2003g:16009.

6. L. W. Christensen, A. Frankild and H. Holm, On Gorenstein projective, injective and flat dimensions - A functorial description with applications, J. Algebra 302(1) (2006), 231-279. MR 2236602.

7. E. E. Enochs and O. M. G. Jenda, Gorenstein injective and projective modules, Math. Z. 220(4) (1995), 611-633. MR 1363858 (97c:16011).

8. E. E. Enochs, O. M. G. Jenda and J. Z. Xu, Foxby duality and Gorenstein injective and projective modules, Trans. Amer. Math. Soc. 348(8) (1996), 3223-3234. MR 1355071 (96k:13010).

9. H.-B. Foxby, Gorenstein modules and related modules, Math. Scand. 31 (1972), 267284. MR 48 \#6094.

10. H.-B. Foxby, Gorenstein dimensions over Cohen-Macaulay rings, in Proceedings of the international conference on commutative algebra (Bruns W., Editor) (Universität Osnabrück, Osnabrück, Germany, 1994), 59-63.

11. A. Frankild and S. Sather-Wagstaff, Reflexivity and ring homomorphisms of finite flat dimension, Commun. Algebra 35(2) (2007), 461-500. MR 2294611.

12. E. S. Golod, $G$-dimension and generalized perfect ideals, in Algebraic geometry and its applications, vol. 165 (Trudy Mat. Inst. Steklova, 1984), 62-66, MR 85m:13011.

13. R. Hartshorne, Residues and duality, Lecture Notes in Mathematics, vol. 20 (SpringerVerlag, Berlin, 1966). MR 36 \#5145.

14. M. Hochster and Y. Yao, An embedding theorem for modules of finite (G-)projective dimension, preprint (2009).

15. H. Holm and P. Jørgensen, Semidualizing modules and related Gorenstein homological dimensions, J. Pure Appl. Algebra 205(2) (2006), 423-445. MR 2203625.

16. H. Holm and D. White, Foxby equivalence over associative rings, J. Math. Kyoto Univ. 47(4) (2007), 781-808. MR 2413065.

17. S. Sather-Wagstaff, Bass numbers and semidualizing complexes, in Commutative algebra and its applications (Walter de Gruyter, Berlin, 2009), 349-381. MR 2640315.

18. S. Sather-Wagstaff, T. Sharif and D. White, Tate cohomology with respect to semidualizing modules, J. Algebra 324(9) (2010), 2336-2368. MR 2684143.

19. R. Takahashi and D. White, Homological aspects of semidualizing modules, Math. Scand. 106(1) (2010), 5-22. MR 2603458.

20. W. V. Vasconcelos, Divisor theory in module categories (North-Holland Publishing Co., Amsterdam, 1974), North-Holland Mathematics Studies, No. 14, Notas de Matemática No. 53. [Notes on Mathematics, No. 53]. MR 0498530 (58 \#16637).

21. D. White, Gorenstein projective dimension with respect to a semidualizing module, J. Commut. Algebra 2(1) (2010), 111-137. MR 2607104. 